\title{
Do DSM-5 Eating Disorder Criteria Overpathologize Normative Eating Patterns among Individuals with Obesity?
}

\author{
Jennifer J. Thomas, ${ }^{1,2}$ Katherine A. Koh, ${ }^{2}$ Kamryn T. Eddy, ${ }^{1,2}$ Andrea S. Hartmann, ${ }^{3}$ \\ Helen B. Murray, ${ }^{1}$ Mark J. Gorman, ${ }^{2,4}$ Stephanie Sogg, ${ }^{2,4}$ and Anne E. Becker ${ }^{1,2,5}$ \\ ${ }^{1}$ Eating Disorders Clinical and Research Program, Massachusetts General Hospital, Boston, MA 02114, USA \\ ${ }^{2}$ Department of Psychiatry, Harvard Medical School, Boston, MA 02114, USA \\ ${ }^{3}$ Institute for Psychology, University of Osnabrück, 49074 Osnabrück, Germany \\ ${ }^{4}$ Massachusetts General Hospital Weight Center, Boston, MA 02114, USA \\ ${ }^{5}$ Department of Global Health \& Social Medicine, Harvard Medical School, Boston, MA 02115, USA
}

Correspondence should be addressed to Jennifer J. Thomas; jjthomas@mgh.harvard.edu

Received 15 February 2014; Accepted 26 May 2014; Published 26 June 2014

Academic Editor: Allan S. Kaplan

Copyright (c) 2014 Jennifer J. Thomas et al. This is an open access article distributed under the Creative Commons Attribution License, which permits unrestricted use, distribution, and reproduction in any medium, provided the original work is properly cited.

Background. DSM-5 revisions have been criticized in the popular press for overpathologizing normative eating patternsparticularly among individuals with obesity. To evaluate the evidence for this and other DSM-5 critiques, we compared the point prevalence and interrater reliability of $D S M-I V$ versus $D S M$-5 eating disorders (EDs) among adults seeking weight-loss treatment. Method. Clinicians $(n=2)$ assigned DSM-IV and DSM-5 ED diagnoses to 100 participants via routine clinical interview. Research assessors $(n=3)$ independently conferred ED diagnoses via Structured Clinical Interview for DSM-IV and a DSM-5 checklist. Results. Research assessors diagnosed a similar proportion of participants with EDs under DSM-IV (29\%) versus DSM-5 (32\%). DSM-5 research diagnoses included binge eating disorder (9\%), bulimia nervosa (2\%), subthreshold binge eating disorder (5\%), subthreshold bulimia nervosa (2\%), purging disorder (1\%), night eating syndrome (6\%), and other (7\%). Interrater reliability between clinicians and research assessors was "substantial" for both DSM-IV ( $\kappa=0.64,84 \%$ agreement) and DSM-5 ( $\kappa=0.63$, $83 \%$ agreement). Conclusion. DSM-5 ED criteria can be reliably applied in an obesity treatment setting and appear to yield an overall ED point prevalence comparable to DSM-IV.

\section{Introduction}

In 2013, DSM-5 reorganized its eating disorder classification system with the objective of enhancing clinical utility. Changes included the revision of diagnostic criteria and merging of feeding and eating disorders into a single chapter [1]. Of particular relevance to individuals with obesity, DSM5 elevated binge eating disorder (BED) from a provisional research diagnosis to a formal diagnostic category. DSM5 also introduced the new term, avoidant/restrictive food intake disorder (ARFID), to replace and extend DSM-IV feeding disorder of infancy and early childhood. Lastly, the residual category (EDNOS) was reconfigured and renamed other specified feeding or eating disorder (OSFED) implemented when a clinician specifies the reason that an individual does not meet full criteria for a specific feeding or eating disorder (e.g., subthreshold BN, subthreshold BED, and night eating syndrome (NES)) - or unspecified feeding or eating disorder (UFED), when full diagnostic criteria are not met but the reason remains unspecified.

Eating disorders and obesity are highly comorbid, and this may be particularly true for presentations recently added to DSM-5. For example, the 12 -month BED prevalence in the National Comorbidity Survey Replication (NCS-R) was just $1.2 \%$ among US adults, but $85 \%$ of those with BED were overweight or obese [2]. Similarly, NES has a point prevalence of $1 \%$ in the general population but affects $6-14 \%$ of individuals seeking treatment related to obesity [3]. Similarly, investigators have recently commented on the changing "weightscape" of BN, in which a growing number of patients 
with $\mathrm{BN}$ are overweight [4]. Moreover, of potential relevance to the clinical presentation of ARFID, a recent study found that $45 \%$ of adults who self-identified as "picky eaters" were overweight or obese [5].

It is important to note that the DSM-5 Eating Disorders Work Group specifically decided not to make obesity a psychiatric diagnosis, stating that "genetic, physiological, behavioral, and environmental factors that vary across individuals contribute to the development of obesity; thus, obesity per se is not considered a mental disorder" (page 1238) [6]. It would therefore be unfortunate if DSM-5 changes inadvertently pathologized normative eating behaviors among individuals with obesity, who already face discrimination in the workplace, health care facilities, educational institutions, the media, and interpersonal relationships [7]. Furthermore, classifying normative eating behavior as pathological could generate unnecessary referrals that would contribute to rising healthcare costs.

Since proposed DSM-5 criteria were posted for public comment in 2010, the field has raised three primary critiques. The most vociferous came from DSM-IV forefather Allen Frances, who warned in his book Saving Normal that BED is a "fake mental disorder" (page 183) representing nothing more than "gluttony" (page 184) [8]. He and psychologist Thomas Widiger expressed concern that BED and other new diagnoses would "go from not currently recognized as mental disorders to become among the most common of the psychiatric disorders, potentially creating false epidemics of misidentified pseudopatients" (page 122) [9]. The claim that BED is a "fake" disorder was not supported in the World Health Organization's recent World Mental Health epidemiological study, which highlighted greater role impairment among individuals with BED compared to healthy controls [10]. However, more research is needed to determine the degree of impairment associated with DSM5 BED specifically. Furthermore, the concern that DSM-5 revisions will cause an avalanche of new BED cases has not received empirical support. For example, in a secondary analysis of NCS-R data comparing 12-month BED prevalence under DSM- $I V$ versus DSM-5, rates increased only from $1.6 \%$ to $1.7 \%$ in women and remained the same in men $(0.8 \%)$ [11]. However, this study did not focus specifically on an overweight population and did not screen for ARFID and DSM-5 OSFED examples, which may be especially prevalent among individuals with obesity.

The second critique was that DSM-5 revisions may do little to reduce the large proportion of eating disorder cases previously relegated to the EDNOS (now OSFED) category [12]. Available data suggest that, while many individuals with DSM-IV EDNOS will be shifted into the major diagnostic categories, a substantial group will still fall into the residual category. For example, in one study of US females with lifetime eating disorders, the majority did not meet criteria for $\mathrm{AN}, \mathrm{BN}$, or $\mathrm{BED}$ and could only be diagnosed with residual eating disorders under both DSM-IV (67.9\% EDNOS) and DSM-5 (53.3\% OSFED) [13]. However, no study has looked specifically at obese populations, where the prevalence of DSM-5 OSFED remains unknown.
A third critique of DSM-5 revisions was that, because DSM-5 introduces new constructs, clinicians could have trouble applying these more complex criteria in routine practice [9]. This concern is reasonable given the history of a low reliability between clinical and research diagnoses for psychiatric disorders. Whereas diagnostic agreement between structured interviews and clinical diagnoses was substantial in a metaanalysis of DSM-IV eating disorders $(\kappa=0.70)$ [14] little is known about interrater reliability under DSM-5. A recent study found fair to substantial ( $\kappa=0.54$ to 0.80$)$ test-retest reliability among researchers for DSM-5 eating disorders [15], and another study found adequate $(\kappa=0.56)$ test-retest reliability for DSM-5 BED among clinicians [16]. However, no study to date has examined the interrater reliability of DSM5 eating disorder diagnoses among clinicians versus research assessors, who may differ in their interpretation of diagnostic criteria [17].

Therefore, the purpose of this study was to evaluate the prevalence and reliability of DSM-5 eating disorder diagnoses among individuals seeking weight-loss treatment. To our knowledge, this is the first study that features a comprehensive interview of DSM-5 eating disorder constructs in an overweight/obese sample, rather than inferring DSM5 diagnoses from $D S M-I V$-based interviews. In contrast to DSM-5 critiques, we hypothesized that (1) the point prevalence of eating disorders would be comparable under $D S M-I V$ and DSM-5; (2) individuals with either a DSM$I V$ or DSM-5 eating disorder diagnosis would have higher levels of psychopathology and impairment in comparison to healthy controls, supporting the convergent validity of DSM-5 revisions; (3) significantly fewer participants would be diagnosed with DSM-5 OSFED compared to DSM-IV EDNOS; and (4) interrater reliability between clinicians and research assessors would be similar under DSM-IV and DSM-5.

\section{Methods}

2.1. Participants. We recruited study participants from a hospital-based weight treatment center in the Northeastern US, which offers multidisciplinary management of overweight and obesity including nutrition counseling, behavioral weight loss, and bariatric surgery. We invited 147 patients consecutively evaluated by one of two participating psychologists from October 2011 to January 2012 to take part. (All patients referred to the weight management center receive a comprehensive psychiatric evaluation as part of routine care. The two participating psychologists conducted evaluations with approximately $80 \%$ of patients referred to the center during this four-month period, with the remainder of patients being evaluated by part-time clinicians or trainees who did not recruit participants for the research study). Of these, $68 \%(N=100)$ took part. Demographic characteristics are presented in Table 1.

2.2. Diagnostic Assessment. After receiving approximately 30 minutes of training on the new criteria by study investigators, clinicians ( $n=2$ Ph.D. psychologists) diagnosed 
TABLE 1: Demographic characteristics of 100 adults seeking weightloss treatment.

\begin{tabular}{lc}
\hline Sex (\%) & \\
Male & $28(28)$ \\
Female & $72(72)$ \\
\hline Mean age (SD) & $45.8(12.0)$ years \\
\hline Ethnicity (\%) & $10(10)$ \\
Hispanic/Latino & $89(89)$ \\
Not Hispanic/Latino & \\
Race (\%) & $2(2)$ \\
American Indian/Alaska Native & $12(12)$ \\
Black/African American & $2(2)$ \\
Asian & $0(0)$ \\
Native Hawaiian/other Pacific Islanders & $81(81)$ \\
White & $41.9(9.1) \mathrm{kg} / \mathrm{m}^{2}$ \\
\hline Mean BMI (SD) & $2(2)$ \\
\hline Obesity class (\%) & $15(15)$ \\
Overweight (nonobese) & $29(29)$ \\
Class I & $54(54)$ \\
Class II &
\end{tabular}

BMI = body mass index.

${ }^{*}$ The percentages do not add to $100 \%$ because 1 participant did not report ethnicity, and 3 participants did not report race.

$D S M-I V$ and DSM-5 eating disorders via routine clinical interview. Research assessors ( $n=3$ Ph.D. psychologists) later independently conferred diagnoses via the Structured Clinical Interview for DSM-IV and a DSM-5 checklist [18] (see Section 2.3.1) during a telephone interview. The research interviews were audio recorded to allow for examination of interrater reliability among research assessors, which was evaluated in 20 randomly selected cases. Subsequently, the research assessor sent a link, via secure email, to an online survey of self-report assessments (see Section 2.3.2) presented though REDCap (an electronic data capturing system) [19]. The Partners Human Research Committee approved the study protocol.

2.3. Measures. We used the following measures to assess the point prevalence of DSM-IV and DSM-5 eating disorders.

\subsubsection{Interview Measures}

Structured Clinical Interview for DSM-IV (SCID-IV), Eating Disorder Module. The SCID-IV is a semistructured interview instrument that assesses DSM-IV Axis I disorders [20]. We used the eating disorders module only. Interrater reliability in the present study was high: $\kappa=0.87$ (almost perfect according to Landis and Koch [21]) with $95 \%$ agreement (i.e., research assessors agreed on the specific eating disorder diagnosis or noncase status in 19 of the 20 cases randomly selected for double coding).

Diagnostic Interview for DSM-5 Feeding and Eating Disorders. This interview-based assessment was created by B. Timothy
Walsh, Chair of the DSM-5 Eating Disorders Work Group, to determine eating disorder presence according to DSM-5 criteria [18]. Interrater reliability within the present study was high: $\kappa=0.87$ (almost perfect according to Landis and Koch [21]) with 95\% agreement (i.e., researcher assessors agreed on the specific eating disorder diagnosis or noncase status in 19 of 20 cases randomly selected for double coding).

2.3.2. Self-Report Measures. We used the following questionnaires to compare eating pathology, general psychopathology, and clinical impairment among participants diagnosed with eating disorders versus those without eating disorders to establish the convergent validity of DSM-5 categories.

Eating Disorder Examination-Questionnaire. The EDE-Q is the self-report version of a standard interview measure of eating disorder psychopathology [22]. The global score represents the severity of attitudinal pathology in four domains: restraint, eating concern, shape concern, and weight concern. Internal consistency of the EDE-Q global score in the present study was 0.89 .

Eating Disorder Inventory-3. The EDI-3 assesses eating pathology and general psychological constructs of potential etiological relevance to eating disorders [23]. Internal consistency of the EDI-3 eating disorder risk subscale (including drive for thinness, bulimia, and body dissatisfaction) in the present study was 0.94 .

Beck Depression Inventory-II (BDI-II). The BDI-II measures depression severity with items that reflect DSM-IV criteria for major depressive disorder [24]. Internal consistency of the BDI-II in the present study was 0.93 .

Clinical Impairment Assessment (CIA). The CIA measures functional impairment associated with eating disorder symptoms in three domains (personal, cognitive, and social) [25]. Internal consistency of the CIA in the present study was 0.95 .

Body Checking Questionnaire (BCQ). The BCQ assesses the nature and frequency of body checking behaviors [26]. It measures checking related to overall appearance, specific body parts, and idiosyncratic checking. Internal consistency of the BCQ in the present study was 0.96.

State-Trait Anxiety Inventory (STAI). The STAI is a commonly used measure of general anxiety symptoms [27]. In the present study, we used only the 20 items assessing trait anxiety. Internal consistency of the STAI trait subscale in the present study was 0.93 .

2.4. Statistical Analyses. We calculated the overall prevalence of DSM-IV BN and EDNOS (including BED) as well as $D S M-5$ BN, BED, ARFID, and OSFED. (We did not include anorexia nervosa, pica, and rumination disorder in overall prevalence estimates because none of these disorders was a focus of clinical attention at the weight-loss clinic. We refer interested readers to a separate paper that details the frequency and characteristics of pica and rumination 
TABLE 2: DSM-IV versus DSM-5 eating disorder diagnoses conferred by structured interviews in an outpatient weight-loss seeking sample.

\begin{tabular}{|c|c|c|c|c|c|}
\hline \multirow{2}{*}{$D S M-I V$ research diagnosis (SCID-IV) } & \multicolumn{5}{|c|}{ DSM-5 research diagnosis } \\
\hline & $\mathrm{BN}$ & BED & OSFED & No eating disorder & Total \\
\hline $\mathrm{BN}$ & 2 & 0 & 0 & 0 & 2 \\
\hline BED & 0 & 8 & 1 & 0 & 9 \\
\hline EDNOS & 0 & 1 & 17 & 0 & 18 \\
\hline No eating disorder & 0 & 0 & 3 & 68 & 71 \\
\hline Total & 2 & 9 & 21 & 68 & 100 \\
\hline
\end{tabular}

$\mathrm{BN}=$ bulimia nervosa, $\mathrm{BED}=$ binge eating disorder, EDNOS = eating disorder not otherwise specified, and OSFED = other specified feeding or eating disorders.

TABLE 3: Psychopathology comparison measured by self-report scales for DSM-IV and DSM-5 between eating disorder and noneating disorder cases.

\begin{tabular}{lcccccc}
\hline \multirow{2}{*}{ Research diagnosis } & \multicolumn{2}{c}{ Eating disorder specific psychopathology [mean (SD)] } & \multicolumn{2}{c}{ General psychopathology [mean (SD)] } \\
& EDE-Q & EDI-3 & CIA & BCQ & BDI-II & STAI \\
\hline DSM-IV & & & & & \\
ED & $3.35(0.84)^{*}$ & $52.52(14.45)^{*}$ & $21.13(9.20)^{*}$ & $56.41(25.14)^{* *}$ & $16.90(8.70)^{* *}$ & $46.79(9.73)^{* *}$ \\
No ED & $2.14(0.99)^{*}$ & $34.10(13.09)^{*}$ & $11.56(10.57)^{*}$ & $38.54(14.81)^{* *}$ & $10.80(9.97)^{* *}$ & $39.10(11.81)^{* *}$ \\
DSM-5 & & & & & \\
ED & $3.27(0.87)^{*}$ & $50.73(14.94)^{*}$ & $19.96(9.51)^{*}$ & $55.31(24.99)^{* *}$ & $16.13(8.71)$ & $46.38(9.43)^{* *}$ \\
No ED & $2.13(0.99)^{*}$ & $34.13(13.33)^{*}$ & $11.69(10.78)^{*}$ & $38.26(14.43)^{* *}$ & $10.90(10.15)$ & $38.96(12.02)^{* *}$ \\
\hline
\end{tabular}

$\mathrm{ED}=$ eating disorder, $\mathrm{EDE}-\mathrm{Q}=$ Eating Disorder Examination-Questionnaire, EDI-3 = Eating Disorder Inventory eating disorder risk subscale, CIA = Clinical Impairment Assessment, $\mathrm{BCQ}=$ Body Checking Questionnaire, BDI-II = Beck Depression Inventory II, STAI = State Trait Anxiety Inventory.

${ }^{*} P<0.0001$ for $t$-test comparing means between ED and no ED, ${ }^{* *} P<0.01$ for $t$-test comparing means between ED and no ED.

behavior in the current sample [28].) To evaluate the critique that applying DSM-5 criteria would increase the overall prevalence of eating disorders, we compared eating disorder prevalence under DSM-IV versus DSM-5 using McNemar's test for dependent proportions. To test our hypothesis that both $D S M-I V$ and DSM-5 eating disorder criteria would identify individuals with higher levels of psychopathology and impairment in comparison to healthy controls, thus supporting the convergent validity of DSM-5 diagnoses, we compared scores on self-report measures of eating and general psychopathology between those who did and did not have an eating disorder using a series of independent sample $t$-tests. To correct for family-wise error, we used a Bonferroni correction, dividing the standard alpha level by six (the number of self-report measures), which provided a more conservative alpha level of 0.0083 . To test our hypothesis that significantly fewer participants would receive a residual eating disorder diagnosis (i.e., EDNOS, OSFED) under DSM-5 compared to DSM-IV, we used a McNemar's test for dependent proportions. Lastly, to evaluate interrater reliability of clinical versus research diagnoses under DSM$I V$ and $D S M-5$, we calculated Cohen's kappa and interpreted it according to Landis \& Koch criteria (0.0-0.20 "poor," 0.21-0.40 “fair," 0.41-0.60 "moderate," 0.61-0.80 “substantial," and 0.81-1.00 "almost perfect") [21].

\section{Results}

3.1. Eating Disorder Prevalence under DSM-IV versus DSM5. Table 2 presents eating disorder research diagnoses under $D S M-I V$ and DSM-5. We did not find a significant difference in overall eating disorder point prevalence (including both formal and residual categories) under DSM-IV versus DSM$5(P=\mathrm{ns})$. Applying DSM- $I V$ criteria, 29 participants $(29 \%)$ met criteria for an eating disorder, including $2 \%(n=2)$ with $\mathrm{BN}, 9 \%(n=9)$ with BED, and $18 \%(n=18)$ with EDNOS. Applying DSM-5 criteria, 32 participants (32\%) met criteria for an eating disorder, including $2 \%(n=2)$ with $\mathrm{BN}, 9 \%(n=$ 9) with BED, and $21 \%(n=21)$ with OSFED. No participants were diagnosed with ARFID.

3.2. Convergent Validity. Table 3 presents means and standard deviations of eating and general psychopathology among individuals diagnosed with either a $D S M-I V$ or a $D S M-5$ eating disorder. Regardless of whether DSM-IV or DSM-5 criteria were applied, individuals diagnosed with an eating disorder had significantly higher scores (consistent with greater psychopathology, risk, or distress/impairment) than individuals not diagnosed with an eating disorder, on the EDE-Q, EDI-3 Eating Disorder Risk subscale and CIA ( $P$ 's < $0.0001)$ as well as the BCQ and STAI ( $P$ 's $<0.01)$. However, individuals with eating disorders had higher BDI-II scores only when DSM-IV $(P=0.005)$, but not DSM-5 $(P=0.014)$, criteria were applied.

3.3. Prevalence of Residual Eating Disorders. Contrary to our hypothesis, the proportion of participants diagnosed with residual eating disorders did not differ significantly under $D S M-I V$ versus $D S M-5$ ( $P=\mathrm{ns})$. Applying $D S M-I V$ criteria, EDNOS (including BED, which was listed as an EDNOS example in DSM-IV) accounted for 93\% $(n=27)$ of eating disorders cases. Applying DSM-5 criteria, OSFED accounted 
TABLE 4: Eating disorder diagnoses conferred by structured interview for DSM-IV (SCID-IV) versus routine clinical interview in an outpatient weight-loss seeking sample.

\begin{tabular}{lccccc}
\hline \multirow{2}{*}{$D S M-I V$ clinician diagnosis } & \multicolumn{5}{c}{$S C I D-I V$ research diagnosis } \\
& BN & BED & EDNOS & No eating disorder & Total \\
\hline BN & 1 & 0 & 0 & 0 & 1 \\
BED & 0 & 4 & 3 & 0 & 7 \\
EDNOS & 1 & 3 & 12 & 4 & 20 \\
No eating disorder & 0 & 2 & 3 & 67 & 72 \\
Total & $\mathbf{2}$ & $\mathbf{9}$ & $\mathbf{1 8}$ & $\mathbf{7 1}$ & $\mathbf{1 0 0}$ \\
\hline
\end{tabular}

$\kappa=0.64,84 \%$ agreement; $\mathrm{BN}=$ bulimia nervosa, $\mathrm{BED}=$ binge eating disorder, and EDNOS = eating disorder not otherwise specified.

for $66 \%(n=21)$ of cases. Of these, $29 \%(n=6)$ had NES, $24 \%$ $(n=5)$ had subthreshold BED, $9 \%(n=2)$ had subthreshold $\mathrm{BN}, 4 \%(n=1)$ had purging disorder, and $33 \%(n=7)$ could not be classified as a specific OSFED example (i.e., OSFEDother).

3.3.1. Characteristics of OSFED-Other Participants. Of the seven OSFED-other participants, two endorsed regular purging with laxatives in the past three months but did not endorse the overvaluation of shape or weight that would qualify them for purging disorder. Two additional participants exhibited persistent overeating (without loss of control) followed by nonpurging compensatory behaviors (e.g., fasting all day, exercising for five hours), thus missing the criteria for BN. Another participant met all criteria for BED except that the loss of control eating took place over a four-hour period (rather than a two-hour period), resembling grazing behavior. A further participant met all criteria for BED except reporting just two (rather than three) of the five associated binge features, and the last participant met all criteria for BED except that he did not explicitly endorse marked distress in the SCID interview (despite self-reporting clinically significant impairment on the CIA). Notably, all seven participants who received research diagnoses of OSFED-other were also identified as eating disorder cases by clinicians.

Lastly, 5\% $(n=5)$ of participants reported consuming at least $25 \%$ of their calories after dinner (potentially consistent with the NES variant characterized by "excessive food consumption after the evening meal," page 354) [1] but were not judged to have either a $D S M-I V$ or a $D S M-5$ eating disorder by either clinicians or research assessors.

\subsection{Interrater Reliability of Clinical and Research Diagnoses.} Tables 4 and 5 present a cross tabulated comparison of clinical and research diagnoses for DSM-IV and DSM-5, respectively. The reliability of overarching $D S M-I V$ categories (i.e., BN, BED, EDNOS, or no eating disorder) was "substantial" according to Landis and Koch [21] $(\kappa=0.64$, $84 \%$ agreement). Individual kappas by disorder are listed in Table 6. Under DSM-5, the reliability of overarching categories (i.e., BN, BED, OSFED, or no eating disorder) was also "substantial" ( $\kappa=0.63,83 \%$ agreement). In contrast, the reliability of DSM-5 residual categories-including both OSFED examples and the OSFED-other designation-was "poor" ( $\kappa=0.15,31 \%$ agreement).

\section{Discussion}

To our knowledge, this is the first study evaluating the point prevalence of DSM-5 eating disorders in an obesity treatment-seeking sample that did not retrospectively apply $D S M-5$ criteria to cases originally diagnosed under DSM$I V$. This design provided a stronger test of the three main critiques of DSM-5 revisions, including the potential overdiagnosis of eating disorders among individuals with overweight/obesity; the continued preponderance of EDNOS; and the risk of reduced interrater reliability in real-world settings.

With regard to the first critique, applying DSM-5 criteria in an obesity treatment setting did not result in a higher than expected prevalence of eating disorders. Indeed, the $32 \%$ prevalence of DSM-5 eating disorders in our weightloss treatment-seeking sample did not differ significantly from the DSM-IV prevalence in the current sample and was comparable to the average DSM-IV eating disorder prevalence of $36 \%$ reported in a recent review of eating pathology among bariatric surgery candidates [29]. Similarly, the point prevalence of BED in our sample was $9 \%$ using both DSM-IV and DSM-5 criteria, which is comparable to previous studies that have found a DSM-IV BED point prevalence between 6 and 14\% in patients seeking obesity treatment [3]. Also of note, none of our participants met criteria for ARFID, despite recent data indicating that a high proportion of self-identified "picky eaters" are overweight or obese [5]. Importantly, supporting convergent validity, DSM5 eating disorder diagnoses were significantly associated with comorbid psychopathology and clinical impairment.

The second main critique of DSM-5 revisions-that they may not substantially reduce the preponderance of EDNOS [12] - received some support in our study. Not only was OSFED the most common DSM-5 eating disorder diagnosis, but also "other" was the most common presentation of OSFED. Some of the OSFED-other presentations we observed in the present study have been named and described previously (e.g., "nonpurging compensatory eating disorder," [30] $n=2$ ), whereas others have not. The preponderance of OSFED observed in the present study is similar to findings from previous studies that have applied DSM-5 criteria retrospectively [13]. Taken together, these data suggest that even a carefully constructed and well thought-out categorical system may be inadequate to fully capture the diversity of eating disorder phenomenology, highlighting the potential benefit of alternative approaches to classification, such as 
TABLE 5: Eating disorder diagnoses conferred by structured interview for DSM-5 field trial versus routine clinical interview in an outpatient weight-loss seeking sample.

\begin{tabular}{|c|c|c|c|c|c|c|c|c|c|}
\hline \multirow{3}{*}{ DSM-5 clinician diagnosis } & \multicolumn{9}{|c|}{$D S M-5$ research diagnosis } \\
\hline & \multirow{2}{*}{$\mathrm{BN}$} & \multirow{2}{*}{ BED } & \multicolumn{5}{|c|}{ OSFED } & \multirow{2}{*}{ No eating disorder } & \multirow{2}{*}{ Total } \\
\hline & & & Sub-BN & Sub-BED & PD & NES & Other & & \\
\hline $\mathrm{BN}$ & 1 & 0 & 0 & 0 & 0 & 0 & 0 & 0 & 1 \\
\hline BED & 0 & 4 & 0 & 1 & 0 & 2 & 1 & 0 & 8 \\
\hline \multicolumn{10}{|l|}{ OSFED } \\
\hline Sub-BED & 0 & 0 & 1 & 2 & 0 & 0 & 0 & 0 & 3 \\
\hline $\mathrm{PD}$ & 1 & 0 & 0 & 0 & 0 & 0 & 1 & 0 & 2 \\
\hline NES & 0 & 1 & 1 & 0 & 1 & 1 & 0 & 1 & 5 \\
\hline Other & 0 & 1 & 0 & 1 & 0 & 0 & 5 & 2 & 9 \\
\hline No eating disorder & 0 & 3 & 0 & 1 & 0 & 3 & 0 & 65 & 72 \\
\hline Total & 2 & 9 & 2 & 5 & 1 & 6 & 7 & 68 & 100 \\
\hline
\end{tabular}

$\kappa=0.63,83 \%$ agreement; $\mathrm{BN}=$ bulimia nervosa, $\mathrm{BED}=$ binge eating disorder, OSFED $=$ other specified feeding or eating disorder, Sub-BN $=$ subthreshold bulimia nervosa, Sub-BED = subthreshold binge eating disorder, $\mathrm{PD}=$ purging disorder, and NES = night eating syndrome.

TABLE 6: Interrater reliability between structured interview and routine clinical interview for DSM-IV and DSM-5 eating disorder diagnoses in an outpatient weight-loss seeking sample.

\begin{tabular}{lccc}
\hline & Kappa & \% Agreement & $\begin{array}{c}\text { Interpretation } \\
\text { (Landis and Koch [21] })\end{array}$ \\
\hline DSM-IV & & & Substantial \\
BN & 0.66 & $99 \%$ & Moderate \\
BED & 0.46 & $92 \%$ & Moderate \\
EDNOS & 0.55 & $86 \%$ & \\
DSM-5 & & & Substantial \\
BN & 0.66 & $99 \%$ & Moderate \\
BED & 0.42 & $91 \%$ & \\
OSFED & & & Moderate \\
Sub-BED & 0.48 & $96 \%$ & Poor \\
PD & -0.01 & $97 \%$ & Poor \\
NES & 0.14 & $91 \%$ & Moderate \\
Other & 0.59 & $94 \%$ & \\
\hline
\end{tabular}

$\mathrm{BN}=$ bulimia nervosa, $\mathrm{BED}=$ binge eating disorder, $\mathrm{EDNOS}=$ eating disorder not otherwise specified, OSFED = other specified feeding or eating disorder, Sub-BED = subthreshold binge eating disorder, $\mathrm{PD}=$ purging disorder, NES = night eating syndrome.

the National Institute of Mental Health's Research Domain Criteria (RDoC) [31].

The third critique of DSM-5-that clinicians would have trouble applying the new criteria in the "real world" [9]was not supported in our study. Rather, results showed moderate to substantial interrater reliability between clinicians and research assessors using DSM-5 criteria, both for eating disorders overall, as well as for BN, BED, and OSFED individually. This represents no change from DSM$I V$ interrater reliability ratings $[14,17]$ and is consistent with previous studies of test-retest reliability for DSM-5 eating disorders $[15,16]$. The only exception to this pattern was for residual eating disorders (i.e., individual OSFED examples), which had relatively poor reliability in the present study. Though the small sample size for our OSFED comparisons highlights the need for cautious interpretation, the low level of interrater agreement may well have been due to the lack of operationalized criteria for specific OSFED presentations. In sum, despite the increased complexity of DSM-5 compared to $D S M-I V$ and the historically low reliability between research and clinical diagnoses [14], the overarching DSM-5 eating disorder categories performed reliably in this naturalistic clinical setting.

An unexpected but interesting observation from the present study was the challenge of distinguishing NES from normative nighttime overeating. Indeed, $6 \%$ of participants overall were assigned a diagnosis of NES, as identified through persistent nocturnal eating. In addition, a further $5 \%$ who did not receive DSM-5 eating disorder diagnoses reported consuming more than $25 \%$ of their calories after dinner. This latter finding is consistent with a communitybased latent class analysis that identified a large category of "nondepressed evening eaters" of variable BMI who reported eating $50 \%$ of daily caloric intake after $7 \mathrm{pm}$, but not after $11 \mathrm{pm}$, and endorsed few comorbid sleep or mood symptoms [32]. Further research on the optimal method of distinguishing between the evening hyperphagia presentation of NES and normative evening overeating is needed. Redefining NES by late-night eating (post-11 pm) rather than just evening eating (post-7 pm) [32] or requiring additional features such as morning anorexia, insomnia, or the belief that one must eat in order to fall asleep [33] may enhance construct validity and reduce the likelihood of overdiagnosis.

Our findings should be interpreted in light of both strengths and limitations. Regarding strengths, while other studies have compared the prevalence of DSM-IV and DSM5 eating disorders by retrospectively applying DSM-5 criteria, this study is the first to prospectively evaluate the validity and reliability of DSM-5 categories in a weight-loss treatment-seeking sample using a DSM-5-based interview. A second strength is that most studies comparing DSM-IV 
and DSM-5 have focused on samples in which participants are already known to have eating disorders, whereas our use of a weight-loss-treatment sample allowed us to ascertain whether applying DSM-5 diagnostic criteria and guidance would misclassify noncases in a nonpsychiatric population. Regarding limitations, the overall sample size was modest, thus limiting power to detect differences in DSM- $I V$ versus DSM-5 prevalence. Further, the low response rate $(68 \%)$ may have introduced selection bias. Moreover, interrater reliability may have been affected by variables other than $D S M$ criteria, such as whether the interview was conducted in-person versus on the telephone, and the small number of clinical $(n=2)$ and research $(n=3)$ assessors. Other limitations relate to the treatment-seeking nature of the sample. For example, bariatric surgery candidates may minimize eating concerns when screening is conducted as part of surgery eligibility, which could have resulted in an underestimation of eating disorder prevalence. Importantly, this limitation is unlikely to have differentially impacted prevalence under DSM-5 versus DSM-IV, which was our primary research question.

Overall, our findings suggest that the revised DSM-5 eating disorder criteria may provide greater clinical utility compared to DSM-IV and are unlikely to result in a higher prevalence of eating disorders. The lack of clarity in defining NES as well as the high prevalence of OSFED indicate that the criteria may need further refinement in order to most effectively capture eating pathology among individuals with obesity. Thus, the application of dimensional measures (as in RDoC), or frequent updates (e.g., DSM-5.1) that treat DSM-5 as a "living document" [34], are worthy of serious consideration.

\section{Conflict of Interests}

The authors declare that they have no conflict of interests regarding the publication of this paper.

\section{Acknowledgment}

This study was funded by Hilda and Preston Davis Foundation.

\section{References}

[1] American Psychiatric Association, Diagnostic and Statistical Manual of Mental Disorders, American Psychiatric Press, Arlington, Va, USA, 5th edition, 2013.

[2] J. I. Hudson, E. Hiripi, H. G. Pope Jr., and R. C. Kessler, "The prevalence and correlates of eating disorders in the national comorbidity survey replication," Biological Psychiatry, vol. 61, no. 3, pp. 348-358, 2007.

[3] W. Milano, M. De Rosa, L. Milano, and A. Capasso, "Night eating syndrome: an overview," Journal of Pharmacy and Pharmacology, vol. 64, no. 1, pp. 2-10, 2012.

[4] C. M. Bulik, M. D. Marcus, S. Zerwas, M. D. Levine, and M. La Via, "The changing "weightscape" of bulimia nervosa," The American Journal of Psychiatry, vol. 169, no. 10, pp. 1031-1036, 2012.
[5] J. E. Wildes, N. L. Zucker, and M. D. Marcus, "Picky eating in adults: results of a web-based survey," International Journal of Eating Disorders, vol. 45, no. 4, pp. 575-582, 2012.

[6] E. Attia, A. E. Becker, R. Bryant-Waugh et al., "Feeding and eating disorders in DSM-5," American Journal of Psychiatry, vol. 170, pp. 1237-1239, 2013.

[7] R. M. Puhl and C. A. Heuer, "Obesity stigma: important considerations for public health," The American Journal of Public Health, vol. 100, no. 6, pp. 1019-1028, 2010.

[8] A. Frances, Saving Normal: An Insider's Revolt Against Out-ofControl Diagnosis, DSM-5, Big Pharma, and the Medicalization of Ordinary Life, HarperCollins, New York, NY, USA, 2013.

[9] A. J. Frances and T. Widiger, "Psychiatric diagnosis: lessons from the DSM-IV past and cautions for the DSM-5 future," Annual Review of Clinical Psychology, vol. 8, pp. 109-130, 2012.

[10] R. C. Kessler, V. Shahly, J. I. Hudson et al., "A comparative analysis of role attainment and impairment in binge-eating disorder and bulimia nervosa: results from the WHO World Mental Health Surveys," Epidemiology and Psychiatric Sciences, vol. 23, pp. 27-41, 2014.

[11] J. I. Hudson, C. E. Coit, J. K. Lalonde, and H. G. Pope Jr., "By how much will the proposed new DSM-5 criteria increase the prevalence of binge eating disorder?" International Journal of Eating Disorders, vol. 45, no. 1, pp. 139-141, 2012.

[12] C. G. Fairburn and Z. Cooper, "Eating disorders, DSM-5 and clinical reality," British Journal of Psychiatry, vol. 198, no. 1, pp. 8-10, 2011.

[13] P. K. Keel, T. A. Brown, J. Holm-Denoma, and L. P. Bodell, "Comparison of DSM-IV versus proposed DSM-5 diagnostic criteria for eating disorders: reduction of eating disorder not otherwise specified and validity," International Journal of Eating Disorders, vol. 44, no. 6, pp. 553-560, 2011.

[14] D. C. Rettew, A. D. Lynch, T. M. Achenbach, L. Dumenci, and M. Y. Ivanova, "Meta-analyses of agreement between diagnoses made from clinical evaluations and standardized diagnostic interviews," International Journal of Methods in Psychiatric Research, vol. 18, no. 3, pp. 169-184, 2009.

[15] R. Sysko, C. A. Roberto, R. D. Barnes, C. M. Grilo, E. Attia, and B. T. Walsh, "Test-retest reliability of the proposed DSM5 eating disorder diagnostic criteria," Psychiatry Research, vol. 196, no. 2-3, pp. 302-308, 2012.

[16] D. A. Regier, W. E. Narrow, D. E. Clarke et al., "DSM-5 field trials in the United States and Canada, part II: test-retest reliability of selected categorical diagnoses," The American Journal of Psychiatry, vol. 170, no. 1, pp. 59-70, 2013.

[17] J. J. Thomas, S. S. Delinsky, S. A. St. Germain et al., "How do eating disorder specialist clinicians apply DSM-IV diagnostic criteria in routine clinical practice? Implications for enhancing clinical utility in DSM-5," Psychiatry Research, vol. 178, no. 3, pp. 511-517, 2010.

[18] B. T. Walsh, Personal communication, February, 2011.

[19] P. A. Harris, R. Taylor, R. Thielke, J. Payne, N. Gonzalez, and J. G. Conde, "Research electronic data capture (REDCap)a metadata-driven methodology and workflow process for providing translational research informatics support," Journal of Biomedical Informatics, vol. 42, no. 2, pp. 377-381, 2009.

[20] M. B. First, R. L. Spitzer, M. Gibbon, and J. B. W. Williams, Structured Clinical Interview For DSM-IV-TR Axis I Disorders, Research Version, Patient Edition. (SCID-I/P), Biometrics Research, New York State Psychiatric Institute, New York, NY, USA, 2002. 
[21] J. R. Landis and G. G. Koch, "The measurement of observer agreement for categorical data," Biometrics, vol. 33, no. 1, pp. 159-174, 1977.

[22] C. G. Fairburn and S. Beglin, "Eating Disorder Examination Questionnaire (EDE-Q 6.0)," in Cognitive Behavior Therapy and Eating Disorders, C. G. Fairburn, Ed., pp. 309-313, Guilford, New York, NY, USA, 2008.

[23] D. M. Garner, Eating Disorder Inventory-3 (EDI-3), Psychological Assessment Resources, Lutz, Fla, USA, 2004.

[24] A. T. Beck, R. A. Steer, and G. K. Brown, Manual For the Beck Depression Inventory, The Psychological Corporation, Harcourt Brace, San Antonio, Tex, USA, 2nd edition, 1996.

[25] K. Bohn, H. A. Doll, Z. Cooper, M. O'Connor, R. L. Palmer, and C. G. Fairburn, "The measurement of impairment due to eating disorder psychopathology," Behaviour Research and Therapy, vol. 46, no. 10, pp. 1105-1110, 2008.

[26] D. L. Reas, M. A. White, and C. M. Grilo, "Body checking questionnaire: psychometric properties and clinical correlates in obese men and women with binge eating disorder," International Journal of Eating Disorders, vol. 39, no. 4, pp. 326-331, 2006.

[27] C. D. Spielberger, R. L. Gorsuch, R. Lushene, P. R. Vagg, and G. A. Jacobs, Manual For the State-Trait Anxiety Inventory, Consulting Psychologists Press, Palo Alto, Calif, USA, 1983.

[28] C. B. Delaney, K. T. Eddy, A. S. Hartmann, A. E. Becker, H. B. Murray, and J. J. Thomas, "Pica and rumination behavior among individuals seeking treatment for eating disorders and obesity," International Journal of Eating Disorders, 2014.

[29] S. Engel and J. E. Mitchell, "Bariatric surgery," in A Clinician's Guide To Binge Eating Disorder, J. Alexander, A. B. Goldschmidt, and D. Le Grange, Eds., pp. 182-192, Routledge, New York, NY, USA, 2013.

[30] H. A. Davis, L. A. Holland, and P. K. Keel, "A preliminary examination of a nonpurging compensatory eating disorder," International Journal of Eating Disorders, vol. 47, no. 3, pp. 239243, 2013.

[31] T. Insel, B. Cuthbert, M. Garvey et al., "Research Domain Criteria (RDoC): toward a new classification framework for research on mental disorders," The American Journal of Psychiatry, vol. 167, no. 7, pp. 748-751, 2010.

[32] R. H. Striegel-Moore, D. L. Franko, D. Thompson, S. Affenito, A. May, and H. C. Kraemer, "Exploring the typology of night eating syndrome," International Journal of Eating Disorders, vol. 41, no. 5, pp. 411-418, 2008.

[33] K. C. Allison, J. D. Lundgren, J. P. O'Reardon et al., “The Night Eating Questionnaire (NEQ): psychometric properties of a measure of severity of the Night Eating Syndrome," Eating Behaviors, vol. 9, no. 1, pp. 62-72, 2008.

[34] H. C. Kraemer, "An idea worth researching: DSM diagnosis of mental disorders as a living document," International Journal of Eating Disorders, vol. 46, no. 5, pp. 412-415, 2013. 


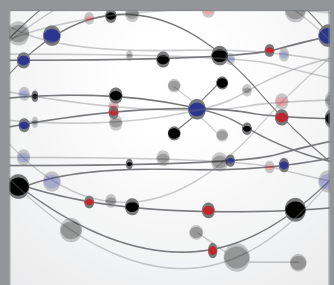

The Scientific World Journal
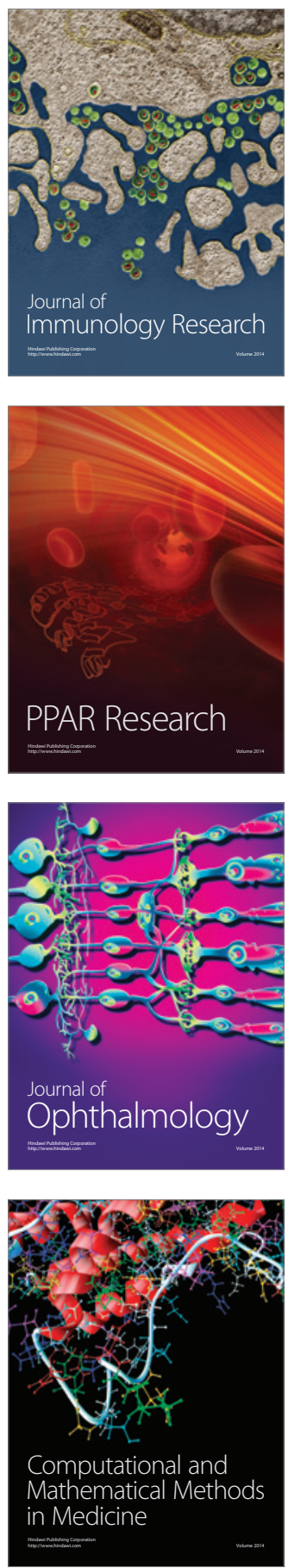

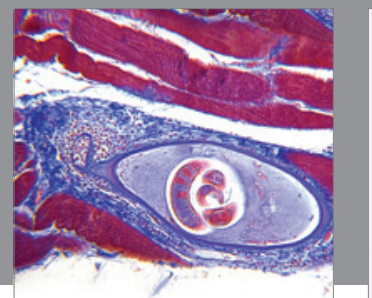

Gastroenterology

Research and Practice
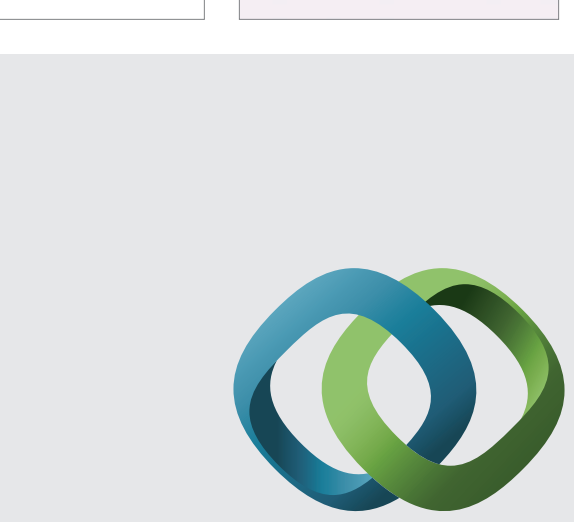

\section{Hindawi}

Submit your manuscripts at

http://www.hindawi.com
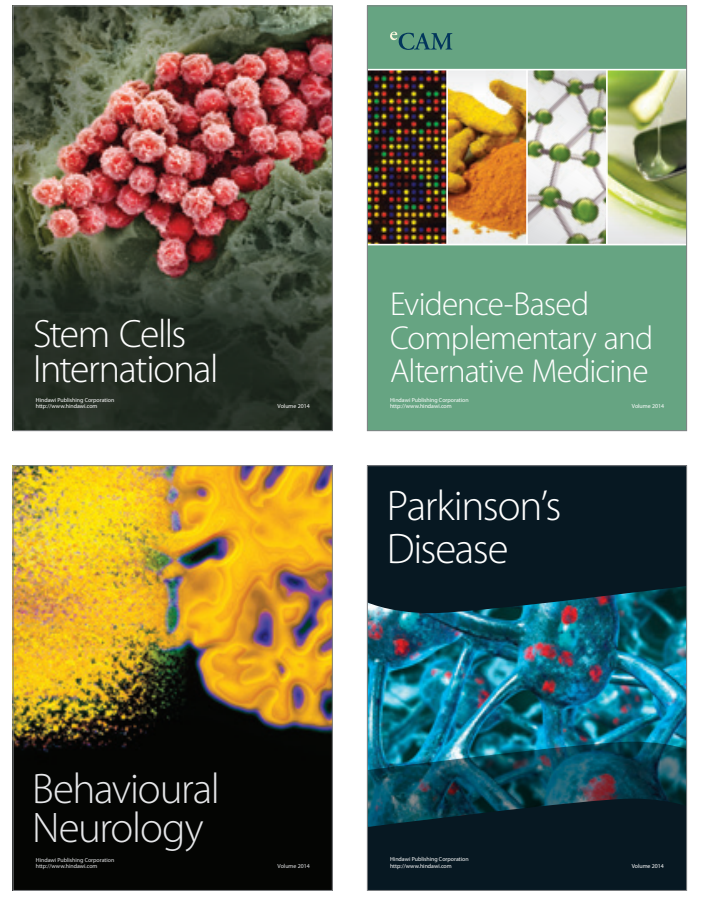
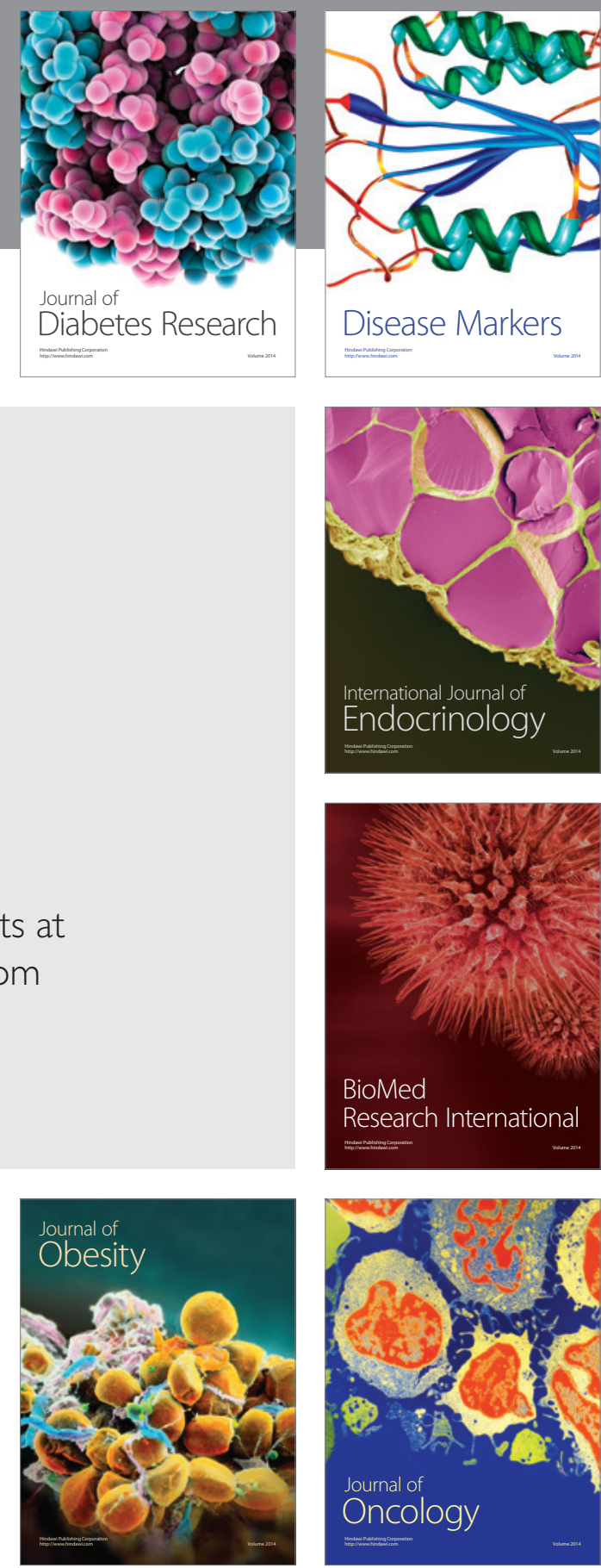

Disease Markers
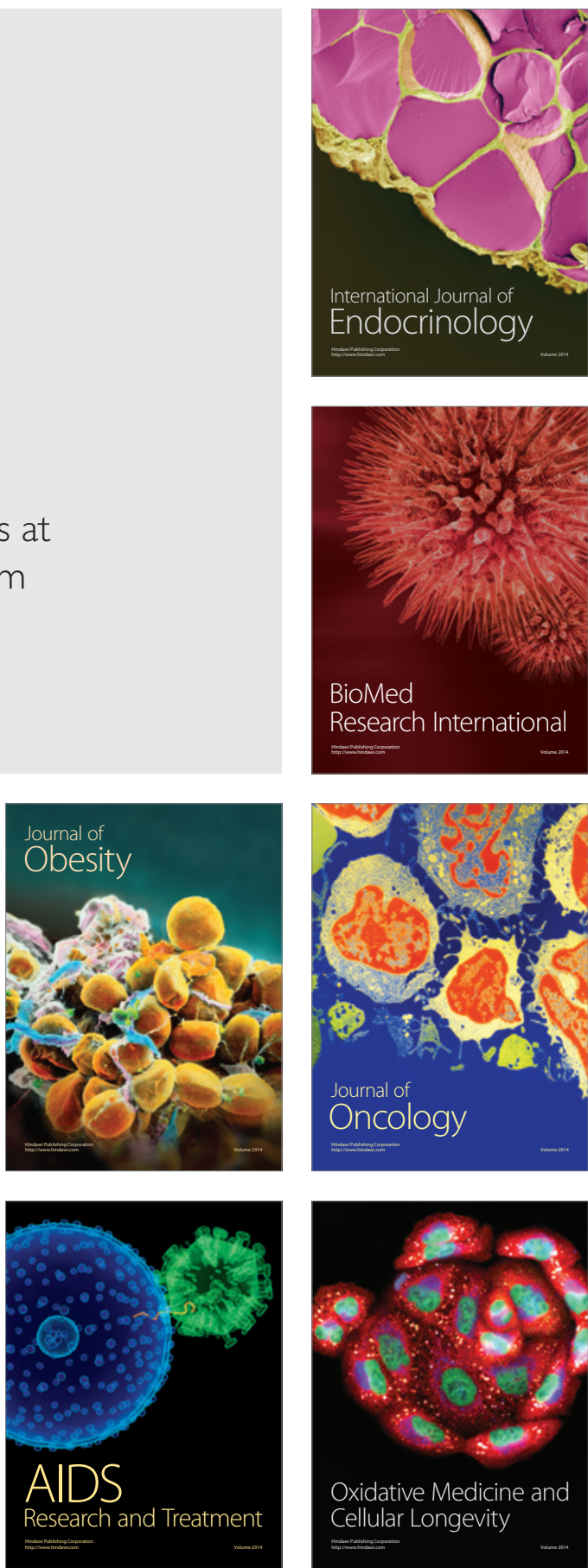